\title{
ANÁLISIS DE INTERFACES CEREBRO-MÁQUINA BASADAS EN IMAGINACIÓN MOTORA DE LA MARCHA
}

\author{
Laura Ferrero, Vicente Quiles, Mario Ortiz, Eduardo Iáñez, José A. Flores, José M. Azorín \\ Brain-Machine Interface System Lab, Universidad Miguel Hernández de Elche, España: 1ferrero@umh.es, \\ vquiles@umh.es,mortiz@umh.es, eianez@umh.es, ja.flores@umh.es,jm.azorin@umh.es
}

\begin{abstract}
Resumen
Las interfaces cerebro-máquina (BMIs de BrainMachine Interfaces) son sistemas que utilizan la actividad cerebral para controlar dispositivos externos. Existen diversos paradigmas de control y uno de los más utilizados se basa en la imaginación motora (IM). La combinación de BMIs basadas en IM con dispositivos de asistencia como exoesqueletos robóticos ofrece nuevas formas de apoyo para pacientes con limitaciones motoras. Además, la realización de IM puede promover mecanismos de plasticidad del sistema nervioso. Este trabajo muestra una revisión de nuestros trabajos previos de BMI no invasivas basadas en IM de la marcha. Se presentan las diversas metodologías utilizadas, las limitaciones de este tipo de BMI y oportunidades de mejora.
\end{abstract}

Palabras clave: Interfaz cerebro-máquina; imaginación motora; señales EEG; exoesqueleto.

\section{INTRODUCCIÓN}

Las interfaces cerebro-máquina (BMIs de BrainMachine Interfaces) son sistemas que utilizan la actividad cerebral para controlar dispositivos externos que pueden ir desde un cursor en una pantalla hasta un dispositivo robótico. El control se basa en la detección de patrones cerebrales [1]. El usuario de la BMI debe alternar entre distintos patrones para producir cambios en el dispositivo externo, lo que se conoce como paradigma de control.

Existen diversos paradigmas de control [1] y uno de los más utilizados es el basado en la imaginación motora (IM). IM se define como la ejecución mental de un movimiento sin activación muscular [2]. Este paradigma se ha empleado para controlar dispositivos de asistencia motora como sillas de ruedas o exoesqueletos robóticos [1].
$\mathrm{Su}$ principal ventaja es su aplicación para la neurorrehablitación. Las terapias tradicionales de rehabilitación se enfocan de forma física a nivel distal. Los terapeutas diseñan ejercicios específicos, observan a los pacientes y manipulan de forma directa los miembros afectados realizando movimientos repetitivos. Estos movimientos se pueden realizar de forma más consistente con dispositivos robóticos. Además, durante el uso de la BMI con IM, el usuario realiza un entrenamiento cognitivo que puede promover la plasticidad del sistema nervioso [1], [3], [4].

Estudios previos han utilizado el contraste entre distintas tareas de IM, como mano derecha/mano izquierda, para producir cambios en los dispositivos externos [5] y otros han contrastado la tarea de IM con un estado inactivo o de relajación [6]. Este trabajo se ha centrado en dos tareas mentales: IM de la marcha y relajación. Uno de los principales problemas de este tipo de BMI reside en las falsas detecciones, es decir cuando el sistema identifica otra tarea mental como IM. En [3] combinan IM con la respuesta inducida por estimulación somatosensorial. En nuestros trabajos previos [7]-[9], el paradigma de IM se combina con un paradigma que mide el nivel de atención del usuario. De esta forma, para conseguir una activación se necesita la realización de IM y mantener un nivel alto de atención.

Como paso previo al control de un exoesqueleto, en nuestro estudio [7], presentamos una BMI basada en IM de la marcha y el nivel de atención para el control de una cinta de andar. Esta BMI se evaluó con 3 sujetos sanos y sin problemas motores. Posteriormente, utilizamos un sistema de realidad virtual con una BMI similar, pero sólo considerando el paradigma de IM y se evaluó con 2 sujetos sanos [8]. En [9] utilizamos la misma BMI que en [7], ajustando los umbrales de activación para controlar un exoesqueleto de miembro inferior y se validó con 2 sujetos sanos. El objetivo de la investigación es el desarrollo de una BMI para el control de un exoesqueleto de miembro inferior que proporcione asistencia y promueva la rehabilitación de pacientes con limitaciones motoras. Además, se busca reducir 
los tiempos de entrenamiento necesarios para calibrar la BMI.

En este trabajo, hacemos una revisión de nuestros trabajos previos de BMI basadas en IM de la marcha. Resumimos la metodología y resultados obtenidos. Finalmente, buscamos las limitaciones de este tipo de BMI y las oportunidades de mejora.

\section{MATERIALES Y MÉTODOS}

\subsection{EQUIPO}

Para el registro encefalográfico (EEG), se utilizó un gorro actiCAP (Brain Products $\mathrm{GmbH}$, Alemania) con 32 electrodos. Los electrodos seguían la distribución 10-10 del sistema internacional. La señal EEG fue amplificada mediante el equipo actiCHamp (Brain Products $\mathrm{GmbH}$, Alemania) y transmitida con la unidad MOVE (Brain Products GmbH, Alemania) de forma remota.

En [7] se empleó una cinta de andar y una unidad de medición inercial Tech-IMU V4 (Technaid, España) para monitorizar el movimiento de los sujetos.

En [8] se utilizó el visor VIVE HTC (HTC, Taiwan) (resolución de $2160 \times 1200,1080 \times 1200$ por ojo, con una frecuencia de actualización de $90 \mathrm{~Hz}$ ), dos estaciones base que monitorizaban la posición exacta del visor (HTC, Taiwan) y el software Steam (Valve, Estados Unidos)

En [9], el exoesqueleto H3 (Technaid, España) se empleó para asistir el movimiento de marcha de los participantes.

La Figura 1 muestra los equipos utilizados en cada investigación.

\section{$2.2 \quad$ PROTOCOLO}

En todos los estudios [7]-[9], las sesiones de experimentación se dividieron en dos partes: entrenamiento y testeo. Durante el entrenamiento, los sujetos debían realizar una serie de tareas mentales, pero el dispositivo a controlar no estaba guiado por la BMI sino por comandos previamente definidos. El número de pruebas de entrenamiento fue distinto en cada estudio. En [7], [9], los participantes alternaron pruebas en las que debían estar estáticos mientras alternaban tareas mentales y otras en las que estaban en movimiento. La información de estas pruebas se utilizó para crear dos tipos de modelos, modelos estáticos y en movimiento que se utilizaron en el testeo. En el caso del entorno de realidad virtual [8], se realizaron pruebas físicamente estáticas, estando durante los periodos de relajación, el entorno virtual estático y durante los periodos de imaginación motora de la marcha, en movimiento.
En la segunda parte de la sesión, los dispositivos fueron controlados mediante la BMI. En [7], [9], cada prueba partía de un estado estático y sólo se utilizaban los modelos estáticos para generar comandos de control. En el momento en que la BMI enviaba un comando de movimiento, tanto para la cinta o el exoesqueleto, se utilizaban los modelos en movimiento. De esta forma el control se estableció como una máquina de estados. En [8], solo se utilizó un tipo de modelo.

En [7], [9] se utilizaron dos paradigmas de control, uno basado en IM y otro basado en el nivel de atención. Por ello, durante las pruebas, los sujetos debían alternar periodos de relajación, con IM de la marcha y con periodos en los que debían realizar operaciones matemáticas. Para el paradigma de IM sólo se consideraron los eventos de relajación e IM y para el paradigma de atención se incluyeron los tres. Mientras que los periodos de relajación y operaciones matemáticas se consideraron como baja atención a la marcha, los periodos de IM se consideraron como alta atención. En [8] sólo se evaluó el paradigma de IM.

\section{$2.3 \quad$ BMI}

El primer paso de la BMI es la adquisición de la señal cerebral, en este caso mediante encefalografía. El análisis de esta señal se realizó en tiempo real, por lo tanto, una vez se adquirió una ventana temporal de datos, estos fueron procesador mientras se adquiría la siguiente ventana y así sucesivamente.

En primer lugar, las señales fueron filtradas mediante un filtro Notch a $50 \mathrm{~Hz}$ para eliminar la contribución de la señal eléctrica y un filtro paso alto para eliminar la componente continua. A continuación, en [7], [9] se aplicó el algoritmo [10] para mitigar la presencia de artefactos oculares y variaciones de la señal. Los siguientes pasos de análisis fueron diferentes para cada paradigma de control.

\subsubsection{PARADIGMA DE IM}

Para extraer características de la señal, se utilizó el algoritmo FBCSP (Filter Bank Common Spatial Patterns) [11]. Como primer paso, se aplicó un banco de filtros paso banda y en este caso se aplicaron 4 filtros: $5-10 \mathrm{~Hz}, \quad 10-15 \mathrm{~Hz}, \quad 15-20 \mathrm{~Hz}, \quad 20-25 \mathrm{~Hz}$. Después, se calculó una transformada espacial para cada señal filtrada con el objetivo de maximizar las diferencias entre clases de tareas mentales.

\subsubsection{PARADIGMA DE ATENCIÓN}

Para el paradigma de atención, se aplicaron dos pasos de pre-procesado adicionales antes de extraer 
características. La señal EEG de cada canal se estandarizó siguiendo el método presentado en [12]. A continuación, se aplicó un filtro Laplaciano para reducir el ruido espacial y aislar la actividad eléctrica de cada electrodo.
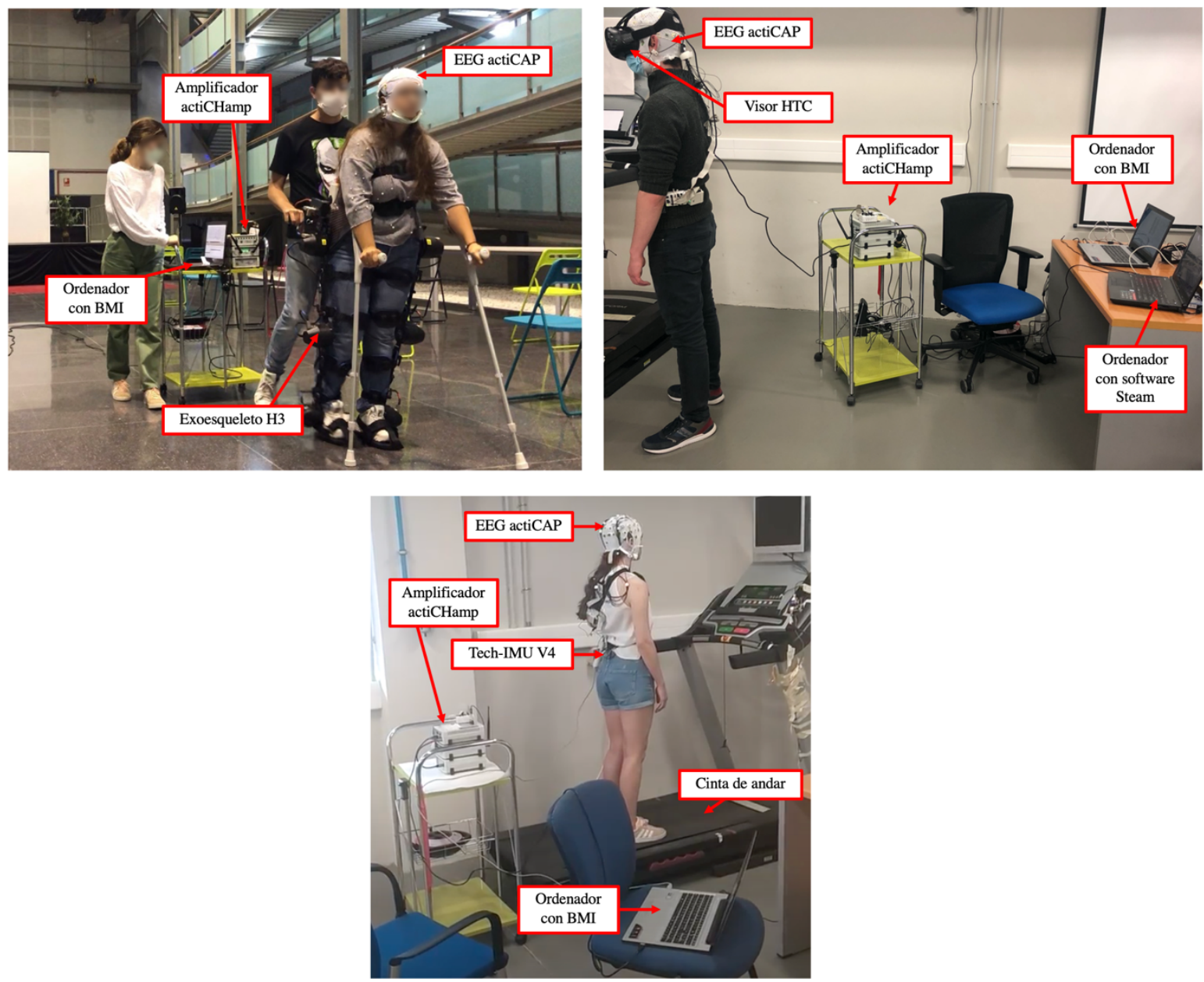

Figura 1: Equipos utilizados en cada investigación: [9] imagen superior izquierda, [8] imagen superior derecha y [7] imagen inferior.

la potencia de la banda gamma de cada electrodo como vector de características. Se escogió esta banda de frecuencia porque está asociada con la concentración [12].

\subsubsection{COMANDOS}

Una vez se extrajeron las características, cada ventana de datos se clasificó mediante el análisis discriminante lineal (LDA de Linear Discriminant Analysis). Las predicciones se promediaron respecto a las últimas 10 ventanas para obtener índices de 0 a 1. En [7], [9] se combinaron los índices del paradigma de IM y del de atención para generar comandos de control.
Se estimó la densidad espectral de potencia mediante MEM (Maximum Entropy Method) [13] y se utilizó 
En promedio, el paradigma de imaginación motora muestra resultados superiores al paradigma de atención. Estos resultados están en consonancia con las conclusiones obtenidas por [6].

También es interesante resaltar que los resultados del paradigma de IM han sido superiores tanto en el lazoabierto como en el lazo-cerrado en el control del entorno de realidad virtual, en comparación con la cinta y el exoesqueleto. Se ha hallado una correlación positiva entre el nivel de inmersión del usuario y su capacidad para controlar un dispositivo externo mediante una BMI [14].

Aunque en la primera sesión no se aprecian diferencias entre las pruebas en estático y las pruebas en movimiento, la precisión del paradigma de IM mejora en las pruebas en estático con las sesiones. Sin embargo, esta evolución favorable con la práctica no se aprecia en las pruebas en movimiento. En estas pruebas, los sujetos debían estar pendientes de no perder el equilibrio y acompañar con las muletas la marcha asistida por el exoesqueleto, a la vez que realizaban una serie de tareas mentales. Es por ello, que podían tener más dificultades para concentrarse.

La mayoría de las investigaciones de BMI basadas en IM se centran en el miembro superior [14], [15], [16] y no en el miembro inferior, debido a la dificultad de registrar la señal EEG durante la marcha [17]. Severens et al. [17] diseñaron una BMI que registraba la actividad cerebral mientras los usuarios realizaban IM de la marcha, estando de pie sobre una cinta de andar. Para el bucle-cerrado, los comandos de control se enviaron a un avatar visual. Los resultados obtenidos en nuestro trabajo previo con una cinta de andar fueron ligeramente superiores [7]. Por otro lado, también hay estudios que utilizaron estímulos externos, como estímulos visuales, para el control de exoesqueletos robóticos de miembro inferior [18]. Aunque los resultados con estos paradigmas de control son superiores a los que utilizan puramente IM de miembro inferior [6], [9], carecen de utilidad desde el punto de vista de la neurrorehabilitación.

\subsection{LIMITACIONES}

Se necesita práctica para el uso de una BMI basada en imaginación motora. Los sujetos deben conseguir modular su actividad cerebral para general distintos comandos de control. Además de la variabilidad que existe entre sujetos, hay quienes no son capaces de realizar esta modulación ni con la práctica. Este fenómeno se conoce como ineficiencia en BMI [19].

Durante el movimiento, existen artefactos producidos por la oscilación de los propios electrodos y por la actividad muscular (EMG). Gran parte de esta interferencia se elimina con los filtros de frecuencia, pero puede quedar alguna componente. Para lidiar con este problema, en [7], [9] se realizaron pruebas en las que el sujeto realizaba distintas tareas mentales, pero siempre en movimiento. De esta forma, si hay algún tipo de artefacto de movimiento, este es común para todas las clases. Sin embargo, sería interesante el uso y desarrollo de algoritmos para eliminar los artefactos de movimiento en tiempo real [20].

Por otro lado, una gran limitación es la complejidad para andar con el exoesqueleto. En [9], los sujetos realizaron una sesión previa en la que practicaron el uso del exoesqueleto antes de utilizar la BMI. Sin embargo, quizá sería interesante incrementar el número de sesiones previas antes de combinar el dispositivo con la BMI.

Como la señal EEG es no estacionaria, el sistema se calibra para cada usuario y sesión, por lo que el tiempo de calibración ocupa gran parte de la sesión. Esto puede producir que los sujetos sufran fatiga y afecte a su concentración y por lo tanto a la eficacia de la BMI [21]. Como posible mejora, se podrían reducir los tiempos de calibración con entrenamientos más efectivos; por ejemplo, mediante sesiones previas con entornos de realidad virtual. Por otro lado, si se diseñasen BMI más generalizadas, se podrían utilizar los datos de sesiones previas para el bucle-cerrado o incluso de otros usuarios [22].

Por último, la motivación de los sujetos parece afectar la eficacia del sistema. Cuando el buclecerrado empezaba sin funcionar correctamente, los usuarios se frustraban $y$ en muchos casos no conseguían mejorarlo. Se ha demostrado una relación entre una motivación externa y la precisión de los resultados [23], pero la influencia del estado emocional aún no se ha podido probar. 
Tabla 1: Resultados en bucle-abierto de las tres investigaciones. Se muestran, por separado, los resultados obtenidos en las pruebas en las que los sujetos estaban en estático y los resultados de las pruebas en movimiento.

\begin{tabular}{|c|c|c|c|c|c|c|c|}
\hline & & & Sesión 1 & Sesión 2 & Sesión 3 & Sesión 4 & Sesión 5 \\
\hline \multirow{4}{*}{ [7] } & \multirow{2}{*}{ Estático } & $\% \mathbf{I M}$ & $73.30 \pm 9.40$ & & & & \\
\hline & & \%Atención & $61.40 \pm 4.90$ & & & & \\
\hline & \multirow{2}{*}{ Movimiento } & $\% \mathrm{IM}$ & $73.70 \pm 3.20$ & & & & \\
\hline & & \%Atención & $66.70 \pm 9.00$ & & & & \\
\hline \multirow{4}{*}{ [9] } & \multirow{2}{*}{ Estático } & $\%$ IM & $56.31 \pm 9.60$ & $69.64 \pm 8.18$ & $65.54 \pm 5.12$ & $64.2 \pm 11.45$ & $74.11 \pm 6.14$ \\
\hline & & \%Atención & $60.67 \pm 7.27$ & $61.70 \pm 8.20$ & $58.21 \pm 8.76$ & $59.52 \pm 8.44$ & $60.83 \pm 5.48$ \\
\hline & \multirow{2}{*}{ Movimiento } & $\% \mathbf{I M}$ & $54.60 \pm 9.78$ & $65.03 \pm 7.27$ & $62.50 \pm 8.91$ & $59.82 \pm 10.11$ & $54.11 \pm 12.71$ \\
\hline & & \%Atención & $63.44 \pm 7.06$ & $66.33 \pm 4.09$ & $61.55 \pm 7.21$ & $65.71 \pm 6.82$ & $70.83 \pm 3.04$ \\
\hline [8] & & $\% \mathrm{IM}$ & $83.45 \pm 38.48$ & & & & \\
\hline
\end{tabular}

Tabla 2: Resultados en bucle-cerrado de las tres investigaciones.

\begin{tabular}{|c|c|c|c|c|c|c|}
\hline & & Sesión 1 & Sesión 2 & Sesión 3 & Sesión 4 & Sesión 5 \\
\hline \multirow{6}{*}{ [7] } & RVP & $100.00 \pm 0.00$ & & & & \\
\hline & $\mathrm{FP} / \mathrm{min}$ & $0.90 \pm 0.50$ & & & & \\
\hline & $\% \mathrm{IM}$ & $67.30 \pm 4.30$ & & & & \\
\hline & \%Atención & $53.00 \pm 3.10$ & & & & \\
\hline & \%Comandos & $75.60 \pm 10.30$ & & & & \\
\hline & Ac & $67.90 \pm 15.40$ & & & & \\
\hline \multirow{6}{*}{ [9] } & RVP & $90.00 \pm 28.28$ & $90.00 \pm 31.62$ & $100.00 \pm 0.00$ & $100.00 \pm 0.00$ & $100.00 \pm 0.00$ \\
\hline & $F P / m i n$ & $1.62 \pm 0.80$ & $1.85 \pm 0.89$ & $3.69 \pm 1.26$ & $1.85 \pm 1.03$ & $2.31 \pm 2.31$ \\
\hline & $\% \mathrm{IM}$ & $50.54 \pm 4.83$ & $54.57 \pm 5.11$ & $51.52 \pm 6.81$ & $56.09 \pm 6.99$ & $59.56 \pm 1.95$ \\
\hline & \%Atención & $52.72 \pm 3.53$ & $58.15 \pm 7.69$ & $49.56 \pm 4.52$ & $63.48 \pm 9.18$ & $65.00 \pm 6.49$ \\
\hline & \%Comandos & $61.80 \pm 6.91$ & $59.90 \pm 11.01$ & $69.80 \pm 7.95$ & $64.20 \pm 8.58$ & $67.80 \pm 14.39$ \\
\hline & Ac & $20.00 \pm 14.14$ & $60.83 \pm 36.40$ & $57.00 \pm 13.04$ & $45.00 \pm 44.72$ & $68.00 \pm 31.76$ \\
\hline \multirow{4}{*}{ [8] } & RVP & $100.00 \pm 0.00$ & & & & \\
\hline & $\mathrm{FP} / \mathrm{min}$ & $3.50 \pm 2.62$ & & & & \\
\hline & \%Comandos & $90.94 \pm 6.69$ & & & & \\
\hline & Ac & $74.48 \pm 8.79$ & & & & \\
\hline
\end{tabular}

\section{CONCLUSIÓN}

Este trabajo muestra una revisión de estudios previos con BMI basadas en la IM de la marcha. Estos estudios utilizaron una BMI para controlar distintos dispositivos externos: una cinta de andar, un entorno de realidad virtual y un exoesqueleto. Existen diversas limitaciones para el uso de estos sistemas como la dificultad parar la realización de IM, la pérdida de concentración y la fatiga producida por largos periodos de calibración. Trabajos futuros deberían intentar mantener la atención de los sujetos, reducir los tiempos de entrenamiento y mejorar la calidad se la señal EEG durante el movimiento.

\section{Agradecimientos}

Esta investigación ha sido realizada en el marco del proyecto "Walk - Control de exoesqueletos de miembro inferior mediante interfaces cerebromáquina para asistir a personas con problemas de marcha" (RTI2018-096677-B-I00), financiado por el Ministerio de Ciencia e Innovación (MCIU), la Agencia Estatal de Investigación (AEI) y la Unión Europea a través del Fondo Europeo de Desarrollo 
Regional (FEDER), y en el marco del proyecto "Desarrollo de nuevas interfaces cerebro-máquina para la rehabilitación de miembro inferior" (GV/2019/009), financiado por la Consejería de Innovación Universidades, Ciencia y Sociedad Digital (Generalitat Valenciana) y el Fondo Social Europeo.

\section{English summary}

\section{ANALYSIS OF BRAIN-MACHINE INTRFACES (BMI) BASED ON MOTOR IMAGERY OF THE GAIT}

\begin{abstract}
Brain-Machine Interfaces (BMI) are systems that use brain activity to control external devices. There are different control paradigms and motor imagery (MI) is one of the most employed. The combination of MI based BMI with assistive devices as exoskeletons offers new opportunities to assist patients with motor limitations. In addition, the performance of MI can promote plasticity mechanisms of the neural system. This research shows a review of our previous works with lower-limb MI based BMI. Different methodologies are presented, as well as limitations of this type of BMI and opportunities for improvement.
\end{abstract}

Keywords: Brain-machine interface; BMI; EEG; exoskeleton.

\section{Referencias}

[1] Y. He, D. Eguren, J. M. Azorín, R. G. Grossman, T. P. Luu, and J. L. ContrerasVidal, "Brain-machine interfaces for controlling lower-limb powered robotic systems," J. Neural Eng., vol. 15, no. 2, 2018.

[2] M. Jeannerod, "Mental imagery in the motor context," Neuropsychologia, vol. 33, no. 11, pp. 1419-1432, 1995.

[3] N. Padfield, J. Zabalza, H. Zhao, V. Masero, and J. Ren, "EEG-Based Brain-Computer Interfaces Using Motor-Imagery: Techniques and Challenges," Sensors (Basel)., vol. 19, no. 6, p. 1423, Mar. 2019.

[4] A. Gharabaghi, "What Turns Assistive into Restorative Brain-Machine Interfaces?," Front. Neurosci., vol. 10, p. 456, 2016.

[5] K. K. Ang, Z. Y. Chin, C. Wang, C. Guan, and H. Zhang, "Filter bank common spatial pattern algorithm on BCI competition IV datasets $2 \mathrm{a}$ and 2b," Front. Neurosci., vol. 6, no. MAR, pp. 1-9, 2012.
[6] M. Ortiz, L. Ferrero, E. Iáñez, J. M. Azorín, and J. L. Contreras-Vidal, "Sensory Integration in Human Movement: A New Brain-Machine Interface Based on Gamma Band and Attention Level for Controlling a Lower-Limb Exoskeleton," Front. Bioeng. Biotechnol., vol. 8, p. 735, 2020.

[7] L. Ferrero, V. Quiles, M. Ortiz, E. Iáñez, and J. M. Azorín, "BCI Based on Lower-Limb Motor Imagery and a State Machine for Walking on a Treadmill," Int. IEEE EMBS Conf. Neural Eng., 2020.

[8] L. Ferrero, M. Ortiz, V. Quiles, E. Iáñez, and J. M. Azorín, "Improving Motor Imagery of Gait on a Brain-Computer Interface by Means of Virtual Reality: A Case of Study," IEEE Access, vol. 9, pp. 49121-49130, 2021.

[9] L. Ferrero, V. Quiles, M. Ortiz, E. Iáñez, and J. M. Azorín, "A BMI Based on Motor Imagery and Attention for Commanding a Lower-Limb Robotic Exoskeleton: A Case Study," Applied Sciences, vol. 11, no. 9. 2021.

[10] A. Kilicarslan, R. G. Grossman, and J. L. Contreras-Vidal, "A robust adaptive denoising framework for real-time artifact removal in scalp EEG measurements," $J$. Neural Eng., vol. 13, no. 2, 2016.

[11] K. K. Ang, Z. Y. Chin, H. Zhang, and C. Guan, "Filter Bank Common Spatial Pattern (FBCSP) in brain-computer interface," Proc. Int. Jt. Conf. Neural Networks, pp. 2390 2397, 2008

[12] Á. Costa et al., "Decoding the Attentional Demands of Gait through EEG Gamma Band Features," PLoS One, vol. 11, no. 4, pp. e0154136-e0154136, Apr. 2016.

[13] B. D. Rainford and G. J. Daniell, " $\mu$ SR frequency spectra using the maximum entropy method," Hyperfine Interact., vol. 87, no. 1, pp. 1129-1134, 1994.

[14] J. M. Juliano et al., "Embodiment Is Related to Better Performance on a Brain-Computer Interface in Immersive Virtual Reality: A Pilot Study," Sensors (Basel)., vol. 20, no. 4, p. 1204, Feb. 2020.

[15] S. N. Resalat and V. Saba, "A Study of Various Feature Extraction Methods on a Motor Imagery Based Brain Computer Interface System.," Basic Clin. Neurosci., vol. 7, no. 1, pp. 13-19, Jan. 2016.

[16] F. Škola, S. Tinková, and F. Liarokapis, "Progressive Training for Motor Imagery Brain-Computer Interfaces Using Gamification and Virtual Reality Embodiment," Front. Hum. Neurosci., vol. 13, p. 329, 2019.

[17] M. Severens, M. Perusquia-Hernandez, B. Nienhuis, J. Farquhar, and J. Duysens, 
"Using Actual and Imagined Walking Related Desynchronization Features in a BCI," IEEE Trans. Neural Syst. Rehabil. Eng., vol. 23, no. 5, pp. 877-886, 2015.

[18] J. W. Choi, S. Huh, and S. Jo, "Improving performance in motor imagery BCI-based control applications via virtually embodied feedback," Comput. Biol. Med., vol. 127, p. 104079, 2020.

[19] N. Leeuwis, A. Paas, and M. Alimardani, "Vividness of Visual Imagery and Personality Impact Motor-Imagery Brain Computer Interfaces.," Front. Hum. Neurosci., vol. 15, p. 634748, 2021.

[20] A. Kilicarslan and J. L. C. Vidal, "Characterization and real-time removal of motion artifacts from $\{\mathrm{EEG}\}$ signals," $J$.
Neural Eng., vol. 16, no. 5, p. 56027, Sep. 2019.

[21] M. A. S. Boksem, T. F. Meijman, and M. M. Lorist, "Effects of mental fatigue on attention: An ERP study," Cogn. Brain Res., vol. 25, no. 1, pp. 107-116, 2005.

[22] F. Lotte, "Signal processing approaches to minimize or suppress calibration time in oscillatory activity-based brain-computer interfaces," Proc. IEEE, vol. 103, no. 6, pp. 871-890, 2015.

[23] S. C. Kleih-Dahms, L. Botrel, and A. Kübler, "The influence of motivation and emotion on sensorimotor rhythm-based brain-computer interface performance.," Psychophysiology, p. e13832, May 2021.

\section{(c)(1) (9)} under the terms and conditions of the Creative Commons Attribution CC BY-NC-SA 4.0 license (https://creativecommons.org/licenses/byncsa/4.0/deed.es).
(C) 2021 by the authors. Submitted for possible open access publication 